\title{
Development and characterization of human iPSC-derived myofibroblast-like cells
}

\author{
Suyama $\mathrm{S}^{1 *}$, Ishida $\mathrm{Y}^{1}$, Yoshimura $\mathrm{N}^{1}$, Miwa $\mathrm{H}^{1}$, Minaguchi $\mathrm{M}^{2}$, Kawakami $\mathrm{N}^{2}$, Notoya $\mathrm{M}^{1}$ and $\mathrm{Takahashi} \mathrm{K}^{1}$ \\ ${ }^{1}$ Drug Discovery and Disease Research Laboratory, Shionogi and Co., Ltd., Osaka 561-0825, Japan \\ ${ }^{2}$ Shionogi Techno Advance Research Co., Ltd., Osaka 561-0825, Japan
}

\begin{abstract}
Neural crest cells give rise to the peripheral nervous system, craniofacial tissues, and adult tissue-specific stem cells, which contribute to tissue homeostasis and regeneration. Misregulation of adult tissue-specific stem cells leads to a variety of diseases. Myofibroblasts, which cause fibrosis, are reported to be derived from tissuespecific stem cells, such as mesenchymal stem cells (MSCs). In this study, we report the generation of myofibroblast-like cells from neural crest-like cells derived from feeder-free human induced pluripotent stem cells (hiPSCs), using a novel protocol that could potentially generate sufficient myofibroblast-like cells for drug screening. The hiPSC-derived myofibroblast-like cells were sensitive to transforming growth factor (TGF)- $\beta$ stimulation, similar to human primary renal fibroblasts. TGF- $\beta$ stimulation increased expression of collagen type1a1 in hiPSC-derived myofibroblast-like cells, and this effect was inhibited by treatment with a TGF- $\beta$ inhibitor. These results indicate that hiPSC-derived myofibroblast-like cells could be a useful tool for in vitro antifibrotic drug screening.
\end{abstract}

\section{Introduction}

a-Smooth muscle actin (aSMA)-positive myofibroblasts are important for tissue regeneration. Tissue-resident fibroblasts and Mesenchymal Stem Cells (MSCs) differentiate into myofibroblasts to repair tissues [1]. However, myofibroblasts are also observed in pathological conditions, such as fibrosis [2]. aSMA-positive myofibroblasts are a major cause of fibrosis, and their level increases with fibrotic disease development [3]. Myofibroblasts are activated by transforming growth factor (TGF) $-\beta$ and produce extracellular matrix (ECM) proteins, including collagen type la1 (Colla1), at a high rate [4].

The origin of tissue-resident fibroblasts differs among tissues. It was reported that lung-resident fibroblasts are derived from hematopoietic stem cells, while hepatic stellate cells (liver-resident fibroblasts) are derived from MSCs [5]. This study compared renal-resident fibroblasts, which are derived from neural crest cells (NCCs) [6] and myofibroblastlike cells, which are obtained from human induced pluripotent stem cell (hiPSC)-derived neural crest like cells (NCLCs) [7].

The number of patients with renal fibrosis is increasing, and this condition is expected to become a major public health burden because there is no drug treatment and patients require dialysis [8]. An in vitro drug screening method that mimics the human in vivo phenotype must be developed to identify an antifibrotic drug.

Renal-resident fibroblasts are the main source of myofibroblasts in renal fibrosis [9]. Therefore, human primary fibroblasts are thought to be useful for antifibrotic drug screening [10]. However, these cells are rarely used because they are difficult to obtain in sufficient numbers for high-throughput drug screening without displaying significant variation.

Pluripotent stem cells (PSCs) can be used to solve this problem. Sufficient numbers of cells that are hard to obtain from humans, such as neurons and cardiomyocytes, can be generated using PSCs [11]. Myofibroblast-like cells can be obtained from PSC-derived NCLCs [12]. NCCs differentiate into a variety of types of cells found in the peripheral nervous system, craniofacial tissues, and adult tissue-specific stem cells [13], and also into renal fibroblasts during kidney development. In response to pathological stimuli, such as hypoxia and nephrotoxic agents, these fibroblasts transdifferentiate into myofibroblasts, which cause renal fibrosis [14].

Here, we report the generation of myofibroblasts from hiPSCs for in vitro antifibrotic drug screening. We modified recently reported protocols to differentiate NCLCs from feeder-free hiPSCs (Ff-hiPSCs) [15]. Ff-hiPSCs can be easily cultured and expanded [16], thereby enabling the generation of sufficient quantities of cells for screening. We then directly differentiated NCLCs into myofibroblast-like cells [12]. hiPSC-derived myofibroblast-like cells were positive for aSMA, FSP-1 (S100A4), and 5'-ectonucleotidase (CD73), which are expressed in myofibroblasts in vivo and commercially available human primary renal fibroblasts [17-19]. Moreover, TGF- $\beta$ stimulation increased Colla1 expression in these cells, and treatment with the TGF- $\beta / S m a d$ inhibitor SB431542 inhibited this effect in a dose-dependent manner. Together, these results suggest that hiPSC-derived myofibroblast-like cells are useful for antifibrotic drug screening.

\section{Materials and methods}

Culture of hiPSCs: This study used 201B7 hiPSCs. Of-hiPSCs were maintained on mouse fetal fibroblasts in ReproStem medium (ReproCELL) supplemented with $5 \mathrm{ng} / \mathrm{ml}$ recombinant human fibroblast growth factor (FGF) 2 (ReproCELL). Ff-hiPSCs were maintained in iMatrix-551 (Nippi)-coated cell culture plates with StemFit AK03N (Ajinomoto) as described previously [16].

${ }^{*}$ Correspondence to: Satoshi Suyama, Drug Discovery and Disease Research Laboratory, Shionogi and Co., Ltd., Osaka 561-0825, Japan, E-mail: satoshi.suyama@shionogi.co.jp

Received: August 03, 2018; Accepted: August 14, 2018; Published: August 16, 2018 
Differentiation of NCLCs: NCLCs derived from 201B7 Of-hiPSCs were differentiated and cultured in low-attachment culture dishes with $\mathrm{KBM}$ (Kohjin Bio) containing $10 \mathrm{ng} / \mathrm{ml} \mathrm{FGF}$ and $10 \mathrm{ng} / \mathrm{ml}$ epidermal growth factor (EGF). The culture media was exchanged every 2 days. Fourteen days later, cells were transferred to fibronectin (CORNING)coated culture dishes. After 14 days of culture, during which the culture media was exchanged every 2 days, CD271(p75NGFR)/CD57(HNK1) double-positive cells were collected using a MoFlo Astrios instrument (Beckman Coulter) and then cultured in myofibroblast differentiation media. 201B7 Ff-hiPSCs were cultured in 6-well plates coated with iMatrix-551. A total of 10,000 Ff-hiPSCs were sub-passaged and cultured for 4 days in StemFit AK03N. Cells were differentiated in KBM supplemented with $10 \mu \mathrm{M}$ SB431542 (Sigma) and $1 \mu \mathrm{M}$ CHIR99021 (WAKO). The culture media was exchanged every day. Cells were harvested after 7 days and cultured in myofibroblast differentiation media.

Differentiation of myofibroblast-like cells from hiPSCs: hiPSCderived NCLCs were cultured for at least 3 weeks in aMEM (Invitrogen) containing 10\% FBS (GIBCO). The culture media was exchanged every 7 days. Cells were passaged when they were $90 \%$ confluent.

Fluorescence-activated cell sorting (FACS): FACS was performed using a MoFlo Astrios instrument according to the manufacturer's protocol. FACS analyses of cells labeled with isotype controls and without antibodies yielded similar results; therefore, the latter were used as control populations. Cells were harvested using TrypLE Select CTS solution (Life Technologies), rinsed in FACS buffer (KBM containing $1 \%$ bovine serum albumin, $0.5 \times$ B27, 5 mM EDTA, and 100 $\mathrm{U} / \mathrm{ml}$ penicillin-streptomycin), incubated with FACS buffer containing Human TruStain FcX Fc Receptor Blocking Solution (1:20; BioLegend) for $5 \mathrm{~min}$, and then labeled with Alexa Fluor-conjugated primary antibodies for $1 \mathrm{hr}$. The following primary antibodies were used: mouse monoclonal anti-CD271-FITC (1:20; BioLegend), mouse monoclonal anti-CD271-PE (1:20; BioLegend), mouse monoclonal anti-CD57APC (1:20; BioLegend), mouse monoclonal anti-CD73-PE (1:20; BD Pharmingen), and rat monoclonal anti-CD44-PE (1:20; BioLegend). The following primary isotype control antibodies were used: FITCconjugated mouse IgG1 (1:20; BioLegend), PE-conjugated mouse IgG1 (1:20; BioLegend), APC-conjugated mouse IgM (1:20; BioLegend), and PE-conjugated rat IgG2b (1:20; BioLegend). FACS data were analyzed using FlowJo V10 (FLOWJO, LCC).

Real-time PCR: Total RNA was purified from cells using a CellAmp ${ }^{\text {ma }}$ Direct RNA Prep Kit for RT-PCR (Takara) according to the manufacturer's instructions. Expression of aSMA and Collal was normalized against that of GAPDH. The primer sets were purchased from Takara. Real-time PCR was performed in a final volume of 25 $\mu \mathrm{l}$ using a One-Step SYBR ${ }^{\star}$ PrimeScript ${ }^{\mathrm{tw}}$ PLUS RT-PCR Kit (Takara) according to the manufacturer's instructions and the 7500 Real-time PCR System (Applied Biosystems). Each PCR comprised 40 cycles with an annealing temperature of $60^{\circ} \mathrm{C}$.

Immunocytochemistry: Cells were perfusion-fixed with $4 \%$ paraformaldehyde for $30 \mathrm{~min}$, rinsed three times with phosphatebuffered saline, incubated in TNB (Tris- $\mathrm{NaCl}$ blocking) solution (Vector Laboratories) for $20 \mathrm{~min}$, labeled with primary antibodies for 1 $\mathrm{hr}$, and then treated with Alexa Fluor-conjugated secondary antibodies (1:250; Invitrogen) for $1 \mathrm{hr}$ at room temperature. The following primary antibodies were used: mouse monoclonal anti-aSMA (1:200; Dako) and rabbit monoclonal anti-S100A4 (1:200; Abcam).
Statistical analysis: All quantified data are expressed as the mean \pm SD. Two mean values were compared using the unpaired Student's $t$ test. $\mathrm{p}<0.05$ was considered statistically significant.

\section{Results}

Of-hiPSC-derived myofibroblast-like cells possess the same characteristics as human primary renal fibroblasts: Renal fibroblasts/ myofibroblasts originate from NCCs [14], and protocols have been developed to generate myofibroblast-like cells from hiPSCs [12]. These cells may be useful for antifibrotic drug screening. However, it is unknown whether hiPSC-derived myofibroblast-like cells express the same markers as human primary renal fibroblasts.

Human primary renal fibroblasts are commercially available. However, comparison of their datasheets revealed that the marker expression patterns differ among products (Table 1). CD73 and FSP-1 are well-recognized fibroblast-specific markers, and $\alpha \mathrm{SMA}$ is expressed in human primary renal fibroblasts $[4,17-20]$. We investigated whether hiPSC-derived myofibroblast-like cells express these three markers.

Myofibroblast-like cells were differentiated from NCLCs that had been processed by fluorescence-activated cell sorting (FACS) (Figure 1A). In this protocol, on-feeder hiPSCs (Of-hiPSCs; 201B7) were harvested and cultured in suspension. After 14 days, neurospheres were cultured in adherent conditions for another 14 days (Figure 1B), and then CD271/CD57 double-positive NCLCs were sorted. The percentage of CD271/CD57 double-positive cells was low $(7.01 \pm 0.86 \%$, $\mathrm{n}=3$ ) (Figure 1C). The NCLCs were cultured for at least 3 weeks in aMEM containing 10\% fetal bovine serum (FBS) to induce their differentiation into myofibroblast-like cells (Figure 1D). Almost 100\% of Of-hiPSC-derived myofibroblasts expressed CD73 (99.11 $\pm 0.45 \%$, $\mathrm{n}=3$, Figure 1E), $\alpha \mathrm{SMA}$, and FSP-1 (100\%, 148/148 cells were doublepositive, Figure 1E). They also expressed the well-recognized fibroblast markers CD44 and HSP47 (data not shown). Thus, Of-hiPSC-derived myofibroblast-like cells expressed the same markers as human primary renal fibroblasts.

We next investigated whether TGF- $\beta$ stimulation increased expression of fibrotic genes, such as Colla1, in these cells. FBS is reported to contain a variety of growth factors, including TGF- $\beta$. To exclude the effects of TGF- $\beta$ in the media, Of-hiPSC-derived myofibroblast-like cells were cultured without FBS for 3 days before TGF- $\beta$ stimulation (Figure 2A). Starvation culture and TGF- $\beta$ stimulation did not cause morphological changes (Figure $2 \mathrm{~B}, \mathrm{C}$ ). Expression of Collal was significantly increased upon TGF- $\beta$ stimulation, while that of $\alpha \mathrm{SMA}$ was increased, but not significantly (Figure 2D). Collal expression in two lots (Lot. 12-0509 and Lot. 12-1109) of commercially available human primary renal fibroblasts also significantly increased in response to TGF- $\beta$ stimulation, as observed in Of-hiPSC-derived myofibroblast-like cells (Figure 2E, F). However, the effect of TGF- $\beta$ stimulation on $\alpha$ SMA expression varied between these two lots. Expression of aSMA was slightly or significantly increased in Lot. 12-0509 upon TGF- $\beta$ stimulation but was not changed in Lot. 12-1109 (Figure 2E, F). These data indicate that hiPSC-derived myofibroblast-like cells produce the fibrotic protein Col1a1, similar to human primary renal fibroblasts.

Table 1. Expression of markers in commercially available human primary renal fibroblasts

\begin{tabular}{|c|c|c|c|c|c|}
\hline & aSMA & CD73 & FSP-1 & vWF & KRT18 \\
\hline DV Biologics, PB-PU001 & + & & & & \\
\hline AvantiCell, KF-HN-051 & + & + & + & & \\
\hline Cell Biologics, H-6016 & - & & & - & - \\
\hline
\end{tabular}




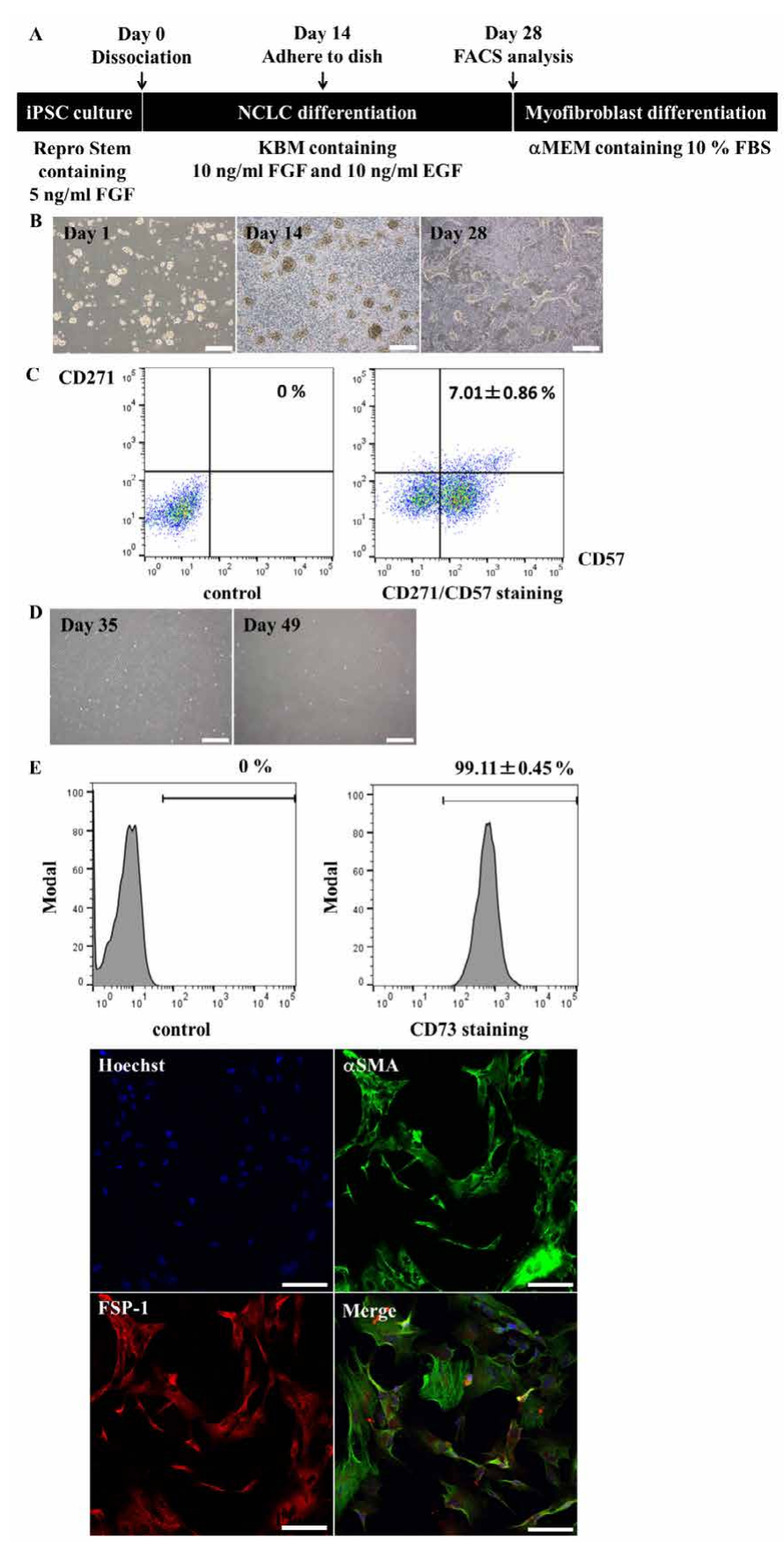

Figure 1. Of-hiPSCs differentiate into myofibroblasts. $A$, Scheme of myofibroblast differentiation. $B$, Morphology of non-attached neurospheres (Day 1 and Day 14) and attached neurospheres (Day 28), which gave rise to NCLCs. Scale bars, $500 \mu \mathrm{m}$. $C$, Quantitative analysis of CD271/CD57 double-positive NCLCs. Values represent the mean $\pm \mathrm{SD}$ (n=3, biological triplicate). $D$, Morphology of myofibroblasts (Day 35 and Day 49). Scale bars, $500 \mu \mathrm{m}$. E, Myofibroblast marker analysis. CD73-positive cells were quantified by FACS, while $\alpha$ SMA- and FSP-1-positive cells were analyzed by immunostaining. Scale bars, $100 \mu \mathrm{m}$. Expression of $\alpha$ SMA and FSP-1 was assessed by determining the percentages of positive cells $(\mathrm{n}=148$ cells). Values represent the mean $\pm \mathrm{SD}(\mathrm{n}=3$, biological triplicate).

Establishment of a protocol to differentiate myofibroblast-like cells from Ff-hiPSCs: Myofibroblast-like cells, which had similar fibrosis-related phenotypes to those of human primary renal fibroblasts, were differentiated from Of-hiPSCs via NCLCs. However, the efficiency of NCLC differentiation was very low (Figure 1C). To overcome this and to develop a protocol for industrial applications, we devised a method to generate myofibroblast-like cells from Ff-hiPSCs. Ff-hiPSCs can be cultured more easily and expanded faster at a lower cost than Of-hiPSCs and are therefore suitable for industrial applications [16].

To overcome this and to develop a protocol for industrial applications, we devised a method, based on a previous protocol [12,21], to generate myofibroblast-like cells from Ff-hiPSCs. Ff-hiPSCs were seeded into a 6-well plate at a density of 10,000 cells/well, expanded over 4 days, and then cultured in F12/DMEM-based KBM Neural Stem Cell media

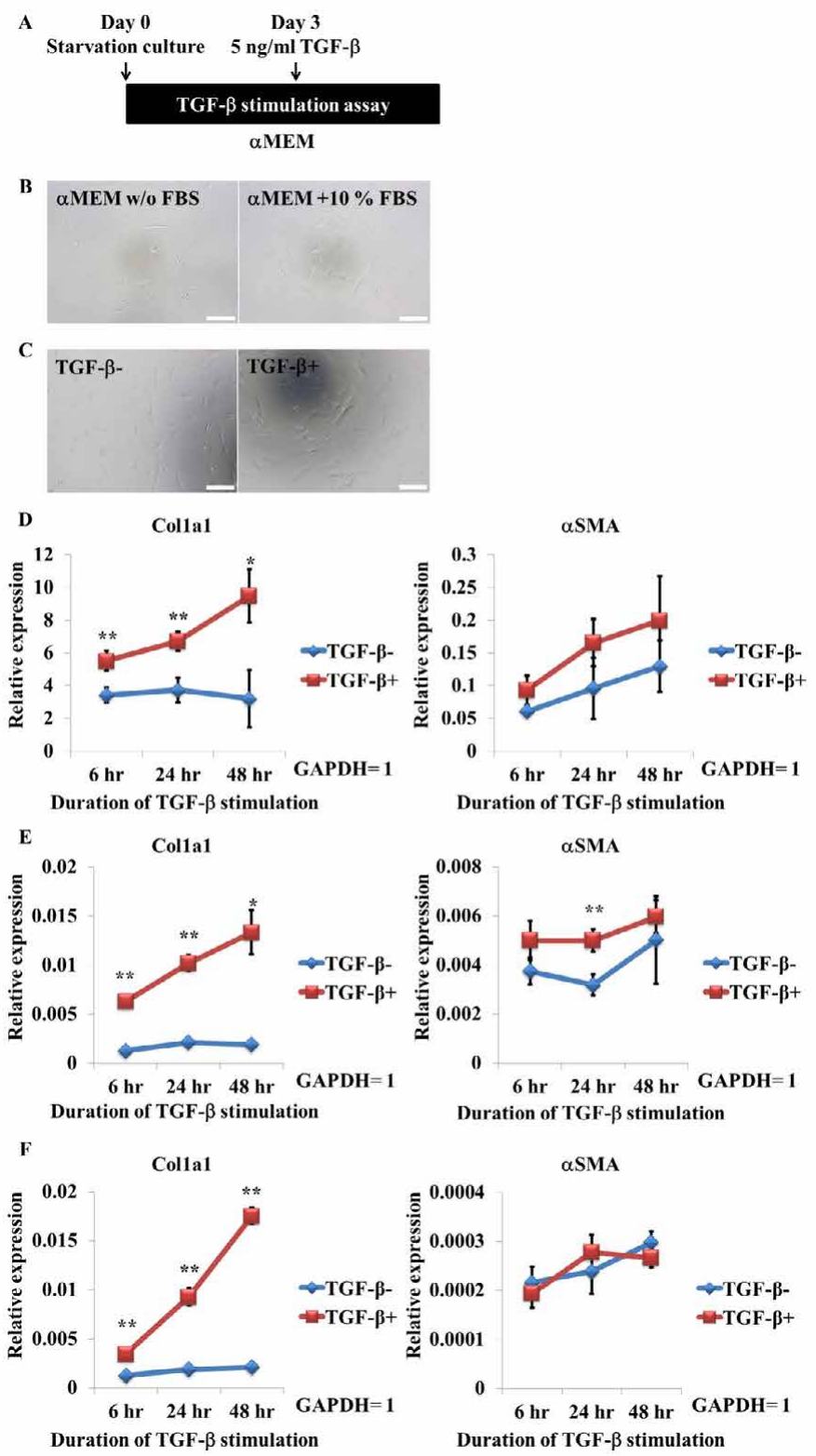

Figure 2. TGF- $\beta$ stimulation increases Colla1 gene expression in Of-hiPSC-derived myofibroblasts and human primary renal fibroblasts. $A$, Scheme of TGF- $\beta$ stimulation. $B$, Morphology of myofibroblasts cultured in $10 \%$ FBS-containing media or FBS-lacking starvation media for 3 days. Scale bars, $200 \mu \mathrm{m}$. C, Morphology of myofibroblasts stimulated with TGF- $\beta$ for $48 \mathrm{hr}$. Scale bars, $200 \mu \mathrm{m}$. $D-F$, Quantitative analysis of Collal and $\alpha$ SMA gene expression in hiPSC-derived myofibroblasts (D) and human primary renal fibroblasts (Lot. 12-0509, E; and Lot. 12-1109, F). Cells were cultured in starvation media for 3 days and then stimulated with TGF- $\beta$. Expression was measured at 6,24 , and $48 \mathrm{hr}$ after TGF- $\beta$ stimulation. Values represent the mean \pm SD. ${ }^{*} \mathrm{p}<0.05$ and ${ }^{* *} \mathrm{p}<0.01$ versus non-stimulated cells $(\mathrm{n}=3$, biological triplicate). 
(KBM) containing $10 \mu \mathrm{M}$ SB431542 and $1 \mu \mathrm{M}$ CHIR99021 (Figure 3A). After 7 days, we observed cell migration using the previously described protocol [21] (Figure 3B). More than 90\% of cells were CD271/CD57 double-positive $(93.81 \pm 1.86 \%, \mathrm{n}=3$ ) (Figure $3 \mathrm{C}$ ). We next investigated whether FACS could be omitted because this procedure can damage cells. The NCLCs were passaged without FACS and cultured for at least 3 weeks in $\alpha$ MEM containing 10\% FBS. There were no morphological differences between myofibroblast-like cells derived from Of-hiPSCs and those derived from Ff-hiPSCs (Figure 3D). Almost 100\% of FfhiPSC-derived myofibroblast-like cells expressed CD73 $(99.23 \pm 0.62 \%$, $\mathrm{n}=3$, Figure $3 \mathrm{E}), \mathrm{aSMA}$, and FSP-1 (100\%, 113/113 cells were doublepositive, Figure 3E), similar to Of-hiPSC-derived myofibroblast-like cells.

We next assessed the fibrosis-related functions of these cells. FfhiPSC-derived myofibroblast-like cells were treated with TGF- $\beta$ in the same way as Of-hiPSC-derived myofibroblast-like cells (Figure 4A). TGF- $\beta$ stimulation did not cause obvious morphological changes in FfhiPSC-derived myofibroblast-like cells (data not shown), similar to our findings in Of-hiPSC-derived myofibroblast-like cells. Expression of Colla1 and aSMA was significantly increased upon TGF- $\beta$ stimulation (Figure 4B). This is consistent with the increase in Collal expression in Of-hiPSC-derived myofibroblast-like cells and human primary renal fibroblasts upon TGF- $\beta$ stimulation (Figure 2D-F).

Treatment with the TGF- $\beta /$ Smad inhibitor SB431542 prevents the increase in Collal expression in TGF- $\beta$-stimulated myofibroblastlike cells: We developed a method to produce sufficient numbers of myofibroblast-like cells for high-throughput drug screening. Using this protocol, $1 \times 10^{4} \mathrm{Ff}$-hiPSCs gave rise to $7.25 \pm 1.56 \times 10^{5}$ myofibroblasts with the same genomic background and no inter-lot variation at day 28 $(n=3)$. We next investigated whether Ff-hiPSC-derived myofibroblastlike cells responded to antifibrotic drugs, such as a TGF- $\beta$ inhibitor, in a concentration-dependent manner using a common antifibrotic drug screening method (Figure 4C). The small molecule SB431542 is a well-known TGF- $\beta /$ Smad inhibitor and is reported to inhibit the fibrotic phenotype [22-24]. SB431542 repressed expression of Colla1 and $\alpha$ SMA in TGF- $\beta$-stimulated Ff-hiPSC-derived myofibroblastlike cells in a concentration-dependent manner (Figure 4D, E). The level of extracellular Colla1 protein, as detected by ELISA, was also reduced by SB431542 (Data not shown). These data demonstrate that Ff-hiPSC-derived myofibroblast-like cells can be used for antifibrotic drug screening.

\section{Discussion}

We demonstrated that hiPSC-derived myofibroblast-like cells exhibited similar fibrosis-related phenotypes as human primary renal fibroblasts. Thus, these hiPSC-derived myofibroblast-like cells may be useful for identifying a new antifibrotic drug.

Fibrotic tissue forms in various organs via wound healing and tissue regeneration [1]. Thus, fibrotic tissue formation is important for homeostasis. However, misregulation of the fibrotic process leads to various diseases, such as chronic kidney disease. Over-proliferation and over-activation of myofibroblasts are observed in such conditions, leading to an increased amount of ECM and loss of organ function.

This study attempted to develop a new tool for studying fibrosis and identifying new drugs using hiPSCs. Myofibroblast-like cells were generated from Of-hiPSC- and Ff-hiPSC-derived NCLCs. The previously reported differentiation efficiency of iPSCs into NCLCs was only $10-80 \%[12,21]$, whereas it was more than $90 \%$ using our
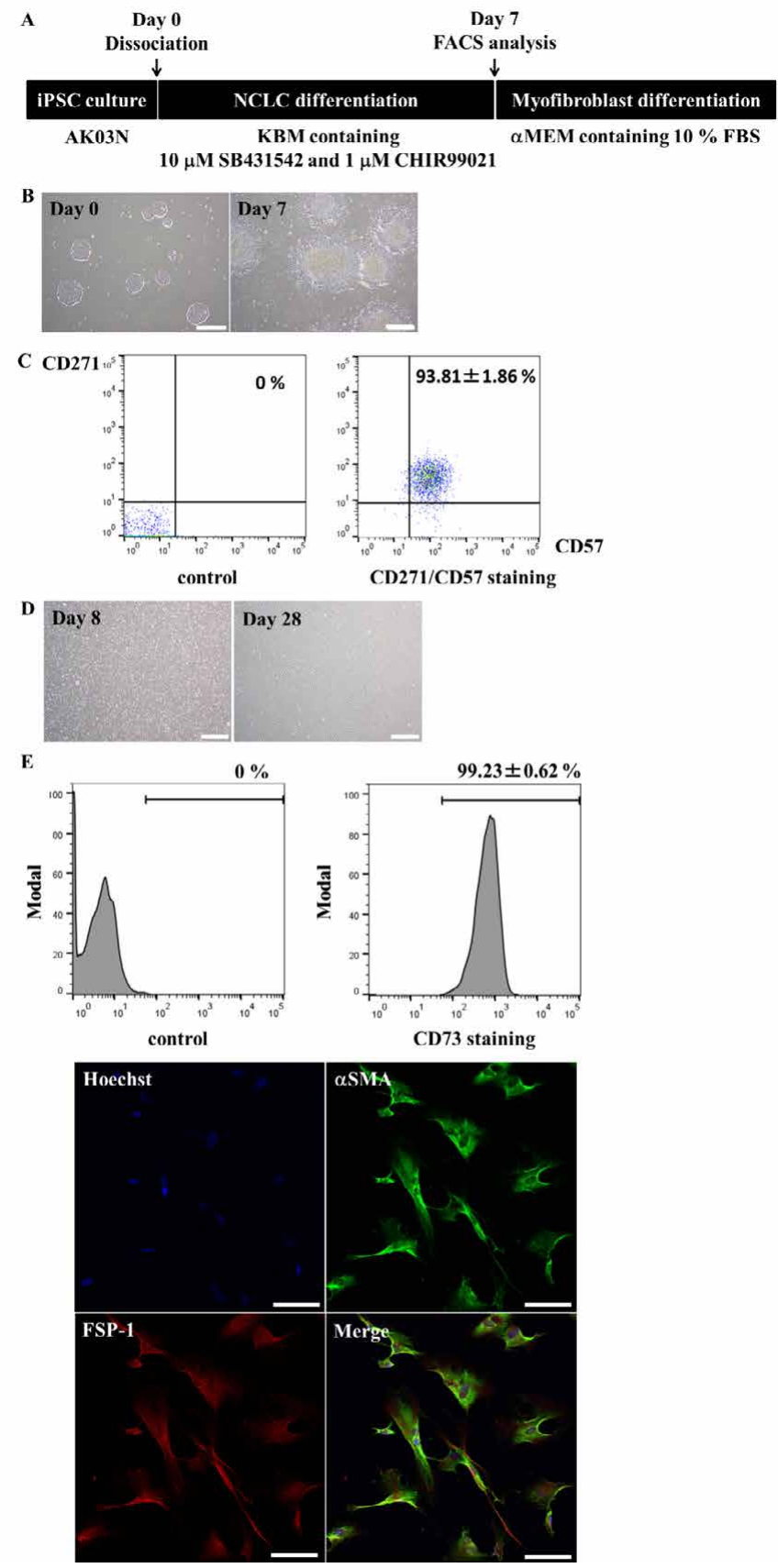

Figure 3. Ff-hiPSCs differentiate into myofibroblasts. $A$, Scheme of myofibroblast differentiation. B, Morphology of cultured cells (Day 0 and Day 7). Scale bars, $500 \mu \mathrm{m}$. $C$, Quantitative analysis of CD271/CD57 double-positive neural crest stem cells. Values represent the mean $\pm \mathrm{SD}(\mathrm{n}=3$, biological triplicate). $D$, Morphology of myofibroblasts (Day 8 and Day 28). Scale bars, $500 \mu \mathrm{m}$. E, Myofibroblast marker analysis. CD73-positive cells were quantified by FACS, while $\alpha$ SMA- and FSP-1-positive cells were analyzed by immunostaining. Scale bars, $100 \mu \mathrm{m}$. Expression of $\alpha$ SMA and FSP-1 was assessed by determining the percentage of positive cells $(\mathrm{n}=113$ cells). Values represent the mean $\pm \mathrm{SD}$ $(n=3$, biological triplicate $)$.

method, suggesting that we had developed an efficient differentiation method to generate NCSCs from hiPSCs.

The hiPSC-derived NCLCs were then used to generate myofibroblast-like cells using a protocol previously used to induce MSCs from hiPSC-derived NCLCs [12,21]. MSCs are a source of myofibloblasts when kidney fibrosis occurs $[3,16]$. In vivo, MSCs are 


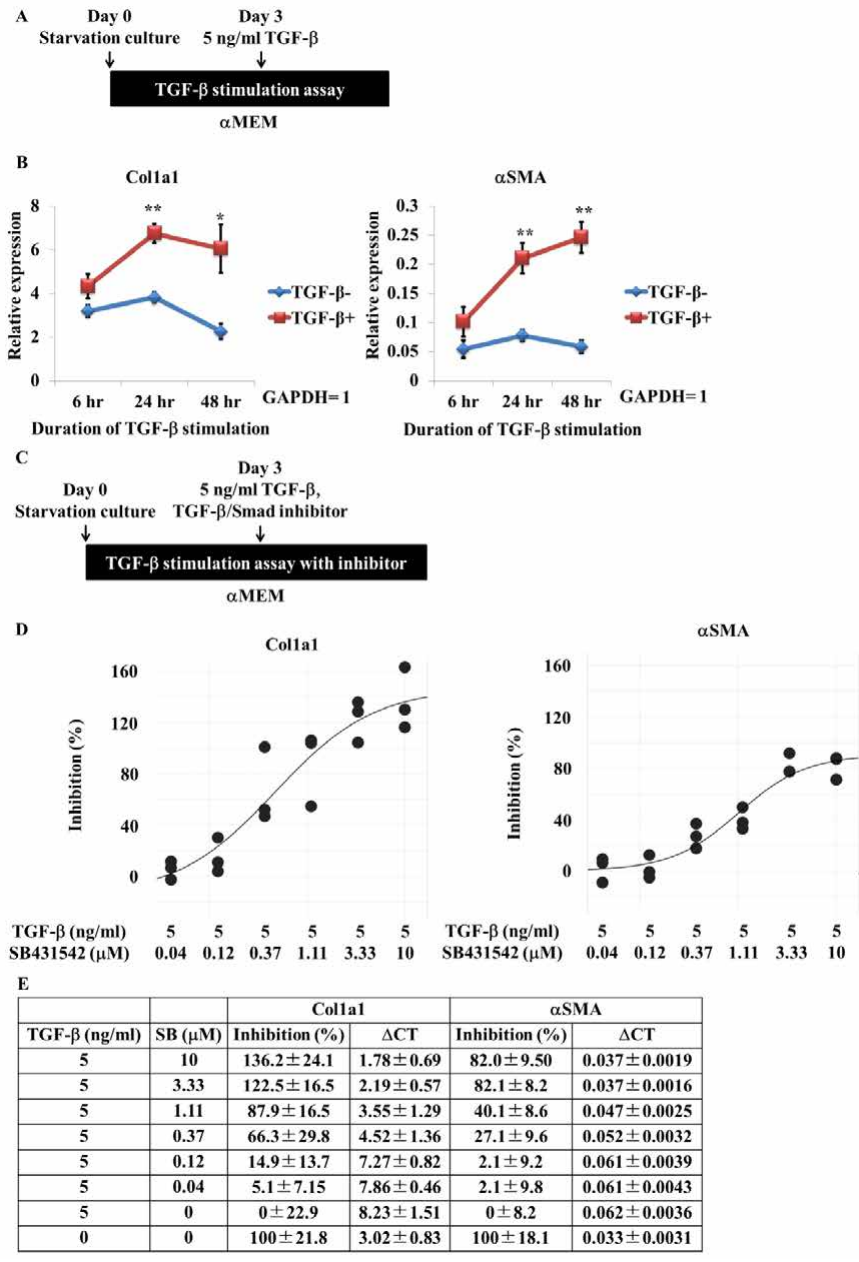

Figure 4. TGF- $\beta$ stimulation increases Colla1 and $\alpha$ SMA gene expression in Ff-hiPSCderived myofibroblasts and is inhibited by the antifibrotic TGF- $\beta /$ Smad inhibitor SB431542. $A$, Scheme of TGF- $\beta$ stimulation. $B$, Quantitative analysis of Colla1 and $\alpha$ SMA expression in hiPSC-derived myofibroblasts. Cells were cultured in starvation media for 3 days and then stimulated with TGF- $\beta$. Expression was measured at 6,24 , and $48 \mathrm{hr}$ after TGF- $\beta$ stimulation. Values represent the mean $\pm \mathrm{SD}$. ${ }^{*} \mathrm{p}<0.05$ and $* * \mathrm{p}<0.01$ versus non-stimulated cells $(\mathrm{n}=3$, biological triplicate). $C$, Scheme of TGF- $\beta$ treatment with/without SB431542. $D$, Percentage inhibition of Collal and $\alpha$ SMA gene expression in TGF- $\beta$-stimulated hiPSC-derived myofibroblasts. Cells were cultured in starvation media for 3 days and then treated with TGF- $\beta$ with/without SB431542. Expression was measured $48 \mathrm{hr}$ later (n=3, biological triplicate). E, Summary of the percentage inhibition data. Values represent the mean $\pm \operatorname{SD}(\mathrm{n}=3$, biological triplicate $)$.

aSMA negative but become positive when subjected to pathological stress signals [1]. MSCs cultured in vitro on stiffness substrates such as plastic dishes that mimic the pro-fibrotic microenvironment differentiate into aSMA-expressing myofibroblasts [23,25]. This may explain why iPSC-derived NCLCs cultured in plastic dishes with MSC differentiation medium automatically differentiated into myofibroblastlike cells and why human primary renal fibroblasts express aSMA during cultivation in vitro but not in vivo.

hiPSC-derived myofibroblast-like cells expressed important markers of human renal fibroblasts, such as aSMA, FSP-1, CD73, CD44, and HSP47 [17-19]. It is unclear whether these markers are sufficient to define the features of myofibroblasts, and we therefore investigated their fibrotic phenotype.

During TGF- $\beta$-stimulated fibrosis, the level of aSMA-positive myofibroblasts increases and these cells secrete Collal into the extracellular space [4,26-28]. Thus, we stimulated hiPSC-derived myofibroblast-like cells with TGF- $\beta$ and compared their levels of Colla1 and $\alpha$ SMA expression with those of commercial human primary renal fibroblasts. Upon TGF- $\beta$ stimulation, expression of Collal was increased in both human iPSC-derived myofibroblast-like cells and human primary renal fibroblasts, whereas expression of aSMA varied between these cells. aSMA expression was increased more in hiPSCderived myofibroblast-like cells than in human primary renal fibroblasts following TGF- $\beta$ stimulation. The level of aSMA expression upon TGF- $\beta$ stimulation might reflect the purity of NCLCs and/or their age. Commercial human primary renal fibroblasts are thought to be more heterogeneous. Indeed, according to their datasheets, $100 \%$ of cells in Lot. 12-0509 and Lot. 12-1109 are CD73-positive, whereas 30.1\% and 93.8\% are FSP-1-positive, respectively, and $70.7 \%$ and $82.3 \%$ are aSMApositive, respectively. This suggests that cell homogeneity underlies the differences in aSMA expression upon TGF- $\beta$ stimulation. Alternatively, differences in age may be responsible. Human primary renal fibroblasts were collected from healthy adults (Lot. 12-0509 is from a 76-year-old Caucasian male, while Lot. 12-1109 is from a 60-year-old black female), while hiPSC-derived cells have fetal characteristics. Expression of Colla1 and aSMA was much higher in hiPSC-derived myofibroblastlike cells than in primary cells. Embryos grow at a fast rate, and NCCs therefore rapidly expand and differentiate into a variety of cell types, including aSMA-positive myofibroblasts, which secrete a large amount of Colla1. However, adult tissue-specific stem cells do not proliferate rapidly or produce a large amount of collagen in the healthy state. This may explain the differences in expression of aSMA and Collal between the cells used in this study.

To investigate whether hiPSC-derived myofibroblast-like cells are useful for drug discovery, they were treated with the antifibrotic compound SB431542. This inhibited acquisition of the fibrotic phenotype in TGF- $\beta$-stimulated cells. It is unknown whether our hiPSC-derived myofibroblast-like cells are exactly the same as human renal fibroblasts. However, hiPSC-derived myofibroblast-like cells exhibited fibrotic phenotypes upon TGF- $\beta$ stimulation, and this was inhibited by treatment with the antifibrotic compound. Moreover, we could generate a large number of myofibroblast-like cells with the same genomic background [29-32]. High-throughput drug screening would be less expensive using these cells than using primary renal-resident fibroblasts. Thus, we believe that hiPSC-derived myofibroblast-like cells can be a useful tool for studying fibrosis and discovering antifibrotic drugs.

\section{Conclusion}

In conclusion, this study demonstrates that hiPSC-derived myofibroblast-like cells can be used to discover antifibrotic drugs. Moreover, these cells may be useful for identifying other drug candidates by elucidating the mechanisms via which stem cells control kidney homeostasis.

\section{Acknowledgment}

We thank the members of the Shionogi laboratory for helpful advice during the course of this study.

\section{Conflicts of Interest}

The authors declare no potential conflicts of interest.

\section{References}

1. Wynn TA, Tamalingam TR(2012) Mechanisms of fibrosis : therapeutic translation for fibrotic disease. Nat Med 18: 1028-1040. 
2. DaSilva Meirelles L, Chagastelles PC, Nardi NB (2006) Mesenchymal stem cells reside in virtually all post-natal organs and tissues. J Cell Sci 119: 2204-2213.

3. Eddy AA (2013) The origin of scar-forming kidney myofibroblasts. Nat Med 19: 964966.

4. Farris AB, Colvin RB (2012) Renal Interstitial Fibrosis: Mechanisms and Evaluation In: Current Opinion in Nephrology and Hypertension. Curr Opin Nephrol Hypertens 21: 289-300.

5. Asahina K, Tsai SY, Li P, Ishii M, Maxson RE, et al. (2009) Mesenchymal origin of hepatic stellate cells, submesothelial cells, and perivascular mesenchymal cells during mouse liver development. Hepatology 49: 998-1011.

6. Davies MR, Liu X, Lee L, Laron D, Ning AY, Kim, et al. (2016) TGF- $\beta$ Small Molecule Inhibitor SB431542 Reduces Rotator Cuff Muscle Fibrosis and Fatty Infiltration By Promoting Fibro/Adipogenic Progenitor Apoptosis. PLoS ONE 11: e0155486.

7. Lee CH, Shah B., Moioli EK, Mao JJ (2010) CTGF directs fibroblast differentiation from human mesenchymal stem/stromal cells and defines connective tissue healing in a rodent injury model. $J$ Clin Invest. 120: 3340-3349.

8. Meguid El Nahas A, Bello AK (2005) Chronic kidney disease: the global challenge. Lancet 365: 331-340

9. LeBleu VS, Taduri G, O'Connell J, Teng Y, Cooke VG, et al. (2013) Origin and function of myofibroblasts in kidney fibrosis. Nat Med 19: 1047-1053.

10. Muller GA, Frank J, Rodemann HP, Engler-Blum G (1995) Human renal fibroblast cell lines (tFKIF and tNKF) are new tools to investigate pathophysiologic mechanisms of renal interstitial fibrosis. Exp Nephrol. 3: 127-133.

11. Takahashi K, Tanabe K, Ohnuki M, Narita M, Ichisaka T, et al. (2007) Induction of pluripotent stem cells from adult human fibroblasts by defined factors. Cell 131: 861872.

12. Lee G, Chambers SM, Tomishima MJ, Studer L (2010) Derivation of neural crest cells from human pluripotent stem cells. Nature Protocols 5: 688-701.

13. Shibata S, Yasuda A, Renault-Mihara F, Suyama S, Katoh H, et al. (2010). Sox10-Venus mice: a new tool for real-time labeling of neural crest linage cells and oligodendrocytes. Mol Brain 3: 31

14. Asada N, Takase M, Nakamura J, Oguchi A, Asada M, et al. (2011) Dysfunction of fibroblasts of extrarenal origin underlies renal fibrosis and renal anemia in mice. J Clin Invest 121: 3981-3990

15. Bajpai R, Chen DA, Rada-lglesias A, Zhang J, Helms J, et al. (2010) CHD7 cooperates with PBAF to control multipotent neural crest formation. Nature 463: 958-962.

16. Nakagawa M, Taniguchi Y, Senda S, Takizawa N, Ichisaka T, et al. (2014). A novel efficient feeder-free culture system for the derivation of human induced pluripotent stem cells. Sci Rep 4: 3594.

17. Strutz F, Okada H Lo, Danoff T, Carone RL (1995) Identification and Characterization of a Fibroblast Marker: FSP1. Journal of Cell Biology 130: 393-405
18. Strutz F, Zeisberg M, Renziehausen A, Raschke B, Becker V, et al. (2001). TGF- $\beta 1$ induces proliferation in human renal fibroblasts via induction of basic fibroblast growth factor (FGF-2). Kidney Int 59: 579-592

19. Strutz F, Zeisberg M (2006) Renal Fibroblasts and Myofibroblasts in Chronic Kidney Disease. J Am Soc Nephrol 17: 2992-2998

20. Sun YBY, Qu X, Garuana G, Li J (2016) The origin of renal fibroblasts/myofibroblasts and signals that trigger fibrosis. Differentiation 92: 102-107

21. Fukuta M, Nakai Y, Kirino K, Nakagawa M, Sekiguchi K, et al. (2014) Derivation of Mesenchymal Stromal Cells from Pluripotent Stem Cells through a Neural Crest Lineage using Small Molecule Compounds with Defined Media. PLoS ONE. 9: e112291

22. Inman GJ, Nicolas FJ, Callahan JF, Harling JD, Gaster LM, et al. (2002) SB-431542 is a potent and specific inhibitor of transforming growth factor-beta superfamily type1 activin receptor-like kinase (ALK) receptors ALK4, ALK5, and ALK7. Mol Pharmacol. 62: 65-74

23. Talele NP, Fradette J, Davies JE, Kapus A, Hinz B (2015) Expression of a-SMA Muscle Actin Determines the Fate of Mesenchymal Stromal Cells. Stem Cell Reports 4: 10161030

24. Yang B, Chen S, Wu M, Zhang L, Ruan M, et al. (2017) PHF14: an innate inhibitor against the progression of renal fibrosis following folic acid-induced kidney injury. $\mathrm{Sci}$ Rep 7: 39888 .

25. Dingal PCDP, Bradshaw AM, Cho S, Raab M, Buxboim A, et al. (2015) Fracta heterogenity in minimal matrix models of scars modulates stiff-niche stem cellresponses via the nuclear exit of a mechnorepressor. Nat Mater 14: 951-960

26. Xu Q, Norman JT, Shrivastav S, Lucio-Cazana J, Kopp JB, et al. (2007) In vitro models of TGF- $\beta$ induced fibrosis suitable for high-throughput screening of antifibrotic agents. Am J Physiol Renal Physiol 293: F631-F640.

27. Kendall RT, Feghali-Bostwick CA (2014) Fibroblasts in fibrosis: novel roles and mediators. Frontiers in pharmacology 5: 123

28. Aoudjehane L, Boelle PY, Bisch G, Delelo R, Paye F, et al. (2016) Development of an in vitro model to test antifibrotic drugs on primary human liver myofibroblasts. Laboratory Investigation 96: 672-79.

29. Achilleos A, Trainor PA (2012) Neural crest stem cells: discovery, properties and potential for therapy. Cell Research 22: 288-304.

30. Avior Y, Sagi I, Benvenisty N(2016) Pluripotent stem cells in disease modelling and drug discovery. Nat Rev Mol Cell Biol 17: 170-182

31. Eckardt KU, Coresh J, Devuyst O, Johnson RJ, Kottgen A, Levey SL, et al. (2013) Evolving importance of kidney disease: from subspecialty to global health burden. Lancet 382: 158-169.

32. McDonald LT, Mehrotra M, LaRue AC (2015) Hematopoietic Origin of Murine Lung Fibroblasts. Stem Cels Int. 159713

Copyright: (C2018 Suyama S. This is an open-access article distributed under the terms of the Creative Commons Attribution License, which permits unrestricted use, distribution, and reproduction in any medium, provided the original author and source are credited. 